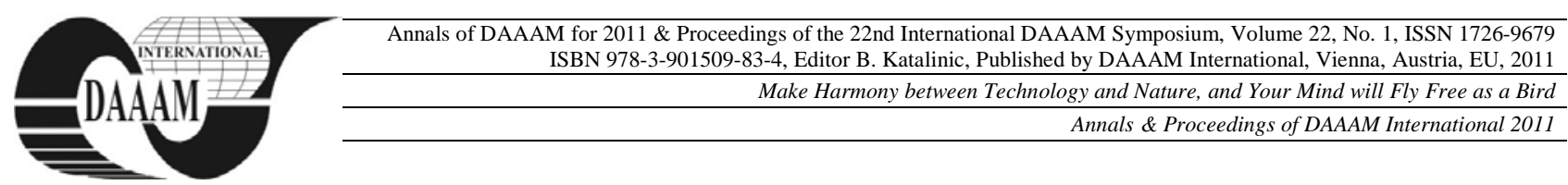

\title{
COMMUNICATION IN VIRTUAL ENTERPRISE PARADIGM
}

\author{
JANUSKA, M[artin]
}

\begin{abstract}
This paper deals with description of eliminating fundamental problems in utilization of virtual enterprise paradigm. The basic problem lies in the lack of mutual confidence and can be greatly alleviated through effective communication. In authors dissertation thesis all problems of the virtual enterprises are addressed trough appropriate communication environment.
\end{abstract}

Key words: communication environment, virtual enterprise, inter firm communication

\section{INTRODUCTION}

Virtual enterprise can be thought of as a project in which number of business subject cooperates. People interested in the project and their ability to communicate with each other effectively is a critical factor for success of the project. Communication between these entities is a source of information and data which all participants need in order to be able to work effectively. Therefore the communication is strongly correlated to the performance. Nowadays there is a significantly increasing pressure on the speed of communication, transparency, relevance and volume of data transmitted.

Virtual enterprise paradigm deals with number of problems during its implementation. Two main problems are lack of mutual confidence between potential partners and no universal communication environment for mutual information exchange.

Main problems in virtual enterprise paradigm are addressed through communication in authors work and dissertation thesis.

Participation in the virtual enterprise means collaboration of a large number of users across a number of totally different business entities. There is none or very minimal possibility that these different entities will unify their enterprise information systems for the short-term cooperation. In the long-term cooperation the idea of unification of corporate information systems in terms of return on investment is also very controversial. Therefore the start of cooperation is threatened by the absence of an effective way of information and data exchange.

Another important question in this concept is traceability and relevance of communication. In terms of trust it is essential that each of the entities is able to monitor and store the communication. This allows reverse analysis of communication back in time.

However, the possibility of reverse analysis is again in terms of cooperation of more SME's inadequate. It is all about quickness, quality and price. Therefore it is necessary to monitor communication and the states of processes in real time. This allows clear identification of problems and greatly eliminates wastage of time in communication. At the same time is evident who is responsible for the waste of time and who will bear the all potential consequences of possible unobserved deadlines. The Ability to monitor communication also applies for the customer who ordered the project. He is therefore given the ability to monitor the state of the communication providing assurance on the progress of work on his projects.

Effective communication environment for affordable price to small and medium-sized enterprises (SME's) are currently on the market only in very limited quantities. There are already existing similar sophisticated massive server-based communication environments or in a smaller scale, electronic forums. However, these solutions require a central server where all data are stored. Paradox is that the concept of communication through a central server as a data repository is very secure and still it meets with the considerable reluctance of companies to store their data on a server which is not directly under their control.

\section{PRINCIPLE OF COMMUNICATION ENVIRONMENT}

Environment works on the basis of email client, which automatically sorts out and stores all received messages. Central data storage is not needed in this concept. The possibility to integrate this client to MS Outlook is still in the progress.

The communication environment for virtual enterprise works on the basis of email client combined with classical electronic forum. For example if somebody in virtual enterprise has a problem that needs to be solved he creates a new topic. This topic is displayed at particular spot in the address structure. During creation the topic gains unique code. First message with this code will be send only to the users which are invited by the creator of this topic. When replying to this message client automatically uses the unique $\mathrm{xml}$ code and sends the reply to all involved users in this topic. Messages are sorted below each other including the enclosures as in classical electronic forum. Any user with sufficient rights can invite another user to this topic and all previous communication will be resend to this newly invited user. This is supposed to help new user to orientate in the problem faster. Creator of the topic can declare it as finished and all content of the topic even with address structure is moved to finished problems section. That helps the communication to stay well sorted and transparent and users can focus on unfinished tasks. During the creation of the topic creator can set deadlines and assign tasks to specific users. Users are informed of approaching deadlines and their tasks in advance. For users with sufficient rights all the information about processing times is available. Each message has in itself encrypted the time when the message was sent. When recipient receives and opens a message automatically another message is send to prove that the message was read. This is important for the project from manager's point of view.

It is essential to mention that communication environment for virtual enterprises works only on email basis. Thus when some meeting or phone call takes place it is necessary to make a record of it and send it via mail to all users. It ensures that project documentation stays compact. 


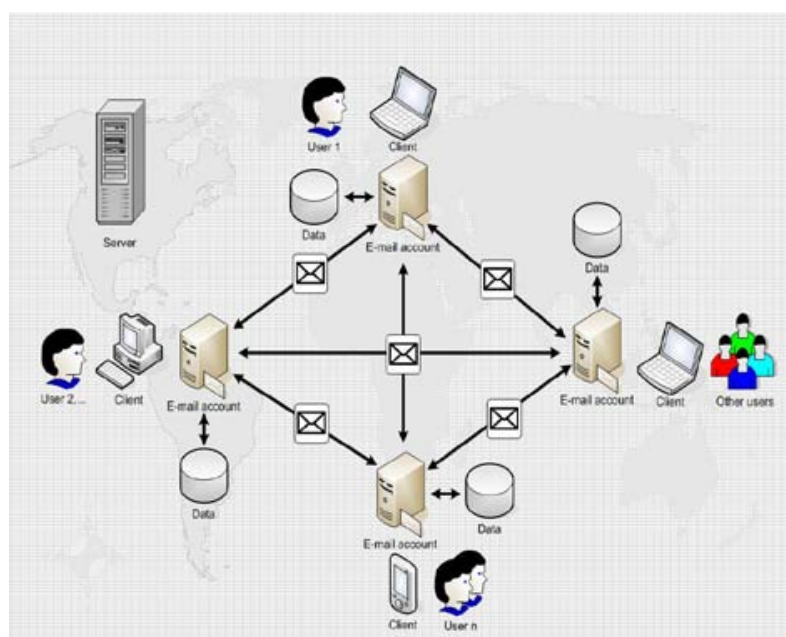

Fig. 1. Communication channels in the environment

As it is possible to see on fig. no.1 all messages have to reach every involved user. When any user opens a message then other information message with time stamp is automatically generated and is send to all users. At the first sight it might seem like senseless overloading of communication channels and making communication less transparent but the opposite is true. By automatically generated messages it is possible to keep track of all information about time and state of the process. By sending information to all involved users we are making sure that all participants on the project have aces to all information. Client is able to sort the messages easily and transparently to the pre-constructed address structure. It is done by the unique encrypted xml code in each message. There is no need for a central server. Users store all the data in their email accounts or on a local drive. Classical solution uses central server are commonly available but during authors interviews with number of company managers author found out that companies do not want to rely on other subjects to store and manipulate with their data. Therefore in this concept everybody keeps its own data and only the emails are being send.

\section{COMMUNICATION STRUCTURE}

Proposed communication environment for virtual enterprises provides the following features addressing communication problems.

\subsection{The communication and information exchange}

a) Creating of clear reporting structure.

b) Ensuring that the message will get to all interested users.

c) Avoiding of unnecessary flooding of unrelated users by unsolicited messages.

d) Assigning tasks to individual users.

e) Environment allows imposing requirements on individual users.

f) Effective feedback.

g) Sorting out of already outdated information.

h) Registering of new users.

i) Posting information on the message board within virtual enterprise.

3.2 Analysis and management of the communication

a) Users with appropriate rights can monitor state of communication and whole process.

b) Allocation of rights to individual users.

c) Reverse analysis of the all time related information related to messaging and tasks.

i.From the perspective of specific message or task.

ii.From the perspective of specific user.

iii.From the perspective of a specific topic or project.

\subsection{Data warehouse}

a) Exchanged data backup.

b) Project documentation export possibility in various data format.

c) Well sorted knowledge base usable in similar projects.

\section{CONCLUSION}

Goal of the research is to design and optimize communication environment for virtual enterprise paradigm support. Specific targets are to make the whole communication traceable well documented and to support larger number of participants across number of companies in better arranged form than classical email client. These advantages are gained by adding the unique xml code to each message which allows the client to sort the messages precisely. Another advantage is the possibility to track and analyze all messages and responsibility for delay back in time in form of Gant charts. Main difference in this solution is no need of central server. According to background search there are no such solutions on the market so far.

It is necessary to ensure the highest possible applicability in real business and therefore sometimes user friendliness and easy control is more important than the complex user functions. Interesting advantages of this concept comparing to the existing solutions are in the easy integration into the company information and communication system. There is no need for the central server, high data security and support of large number of users. Also there is the possibility to transparently sort and track all the messages and perform simple time analyses. This simple and affordable software client provides managers with a relatively strong communication tool with the basic functions for analyzing and managing the communication.

Whole communication environment and communication methodology is described in authors dissertation thesis in detail.

Goals of further research of the author are optimization and further support of virtual enterprise paradigm trough effective communication.

\section{REFERENCES}

Januska, M.; Kurkin, O.; Miller, A. Communication environment for small and medium enterprises In: The 14th International Business Information Management Association Conference, ISBN 978-0-9821489-3-8, International Business Information Management Association (IBIMA) 2010, Istanbul

Januška, M.: Communication environment for virtual enterprise paradigm support, Dissertation thesis, Pilsen: 2010

Kurkin, O.; Šimon, M.;(2010). Optimization of layout using discrete event simulation. In: The 14th International Business Information Management Association Conference, ISBN 978-0-9821489-3-8, International Business Information Management Association (IBIMA) 2010, Istanbul

Januška, M.; Kurkin, O.;(2010). Communication environment designed for project cooperation of small and medium enterprises. In: The 21th International DAAAM SYMPOSIUM "Intelligent Manufacturing \& Automation: Theory, Practice \& Education", ISBN 978-3-901509-73-5, DAAAM International Vienna 2010, Zadar 